\title{
The Consequence of Immune Suppressive Cells in the Use of Therapeutic Cancer Vaccines and Their Importance in Immune Monitoring
}

\author{
Matteo Vergati, Jeffrey Schlom, and Kwong Y. Tsang \\ Laboratory of Tumor Immunology and Biology, Center for Cancer Research, National Cancer Institute, National Institutes of Health, \\ Bethesda, MD 20892, USA \\ Correspondence should be addressed to Jeffrey Schlom, js141c@nih.gov
}

Received 27 October 2010; Accepted 15 December 2010

Academic Editor: Timothy M. Clay

Copyright () 2011 Matteo Vergati et al. This is an open access article distributed under the Creative Commons Attribution License, which permits unrestricted use, distribution, and reproduction in any medium, provided the original work is properly cited.

Evaluating the number, phenotypic characteristics, and function of immunosuppressive cells in the tumor microenvironment and peripheral blood could elucidate the antitumor immune response and provide information to evaluate the efficacy of cancer vaccines. Further studies are needed to evaluate the correlation between changes in immunosuppressive cells and clinical outcomes of patients in cancer vaccine clinical trials. This paper focuses on the role of T-regulatory cells, myeloid-derived suppressor cells, and tumor-associated macrophages in cancer and cancer immunotherapy and their role in immune monitoring.

\section{Introduction}

In April 2010, the U.S. Food and Drug Administration (FDA) approved sipuleucel-T (Provenge, Dendreon Corp., Seattle, WA) for the treatment of asymptomatic or minimally symptomatic metastatic castration-resistant prostate cancer (mCRPC). Provenge has been shown to improve overall survival in mCRPC patients by 4.1 months compared to placebo (25.8 months versus 21.7 months, resp.; $P=.032$; HR: 0.775 ; 95\% CI: $0.614,0.979$ ) [1]. Approval of the first therapeutic cancer vaccine is a milestone in cancer immunotherapy; nevertheless, the question still remains: how do therapeutic cancer vaccines work? Immune response to a pathogen (i.e., virus, bacterium, yeast) or to tumor cells is a complex, incompletely understood process involving multiple factors.

The rationale for therapeutic cancer vaccines (as opposed to preventive cancer vaccines, which are not the subject of this paper) arose from the hypothesis that the cancer cells are under surveillance of a healthy immune system, and that cancer spreads when the host immune system fails to control the growth of tumor cells. The specific reasons for this failure of the immune system are not well known.
In the last decades, much research in cancer immunology has focused on defining tumor-specific antigens or tumorassociated antigens (TAAs) capable of inducing an immune response against tumors. Various vaccine strategies and modalities have also been tested in an effort to achieve this goal $[2,3]$. To date, this pursuit has encountered some major obstacles. The lack of a strong antitumor response might be related to the intrinsic nature of the tumor antigen itself which, unlike a viral or bacterial invader, is usually a self-antigen. Moreover, a weak immune response is frequently associated to the treatment (i.e., chemotherapy or radiotherapy) the cancer patient has previously received. This should thus be taken into account when designing clinical trials employing a combination of cancer vaccines and standard therapies. Particular emphasis should also be placed on the optimal schedule for the various treatments because, while chemotherapy and radiotherapy can have an immunosuppressive effect, studies have shown that they may also increase the expression of several TAAs on tumor cells, or cause a "rebound effect" on immune cells that can be used to enhance the antitumor response (see [3] for review). In this scenario, the analysis of the immunological effects of targeted therapies that use antibodies and small 


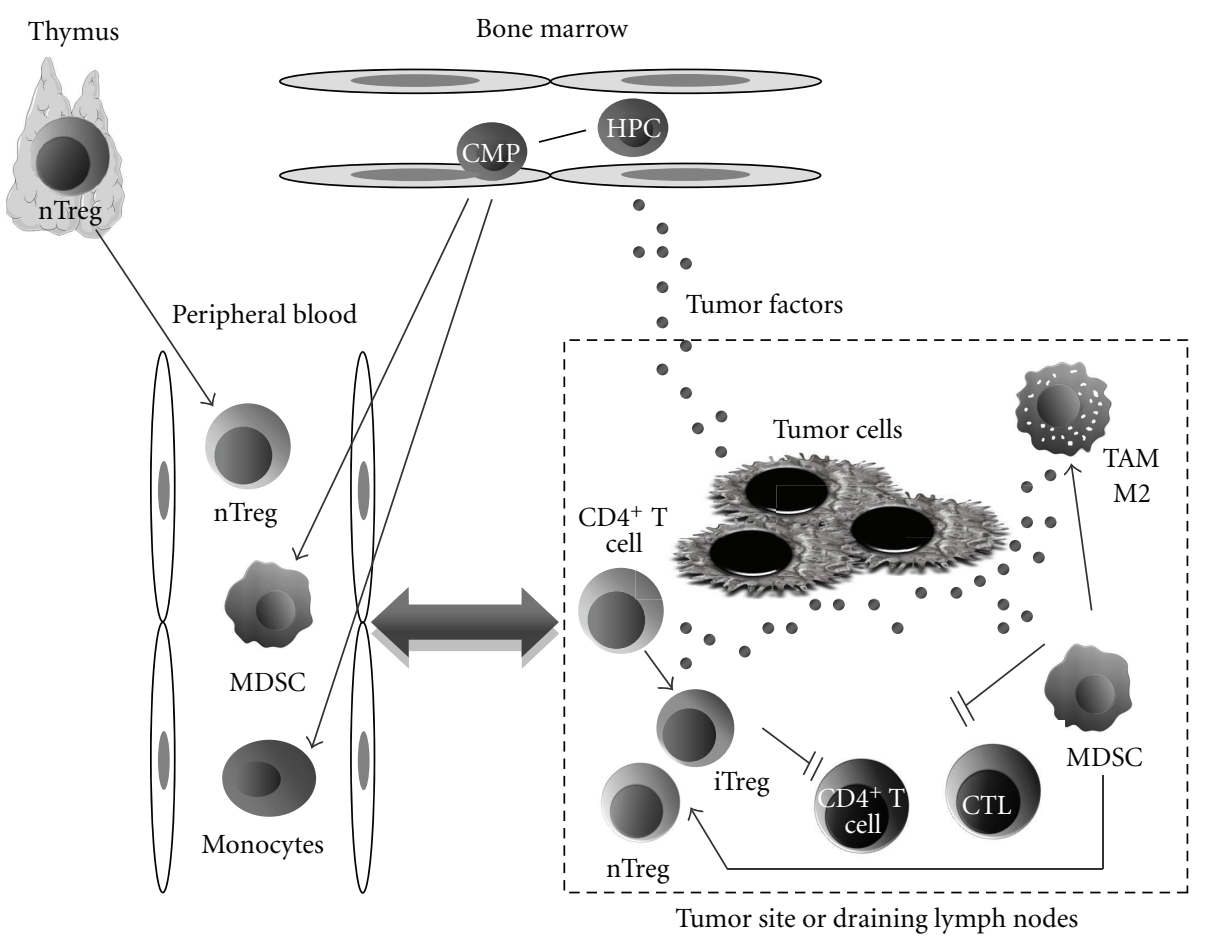

FIGURE 1: Schematic representation of the potential role of the immune suppressive cells (ISCs) in cancer. The release into the circulation of ISCs derived from thymus (nTregs) or from bone marrow (MDSCs) is partially driven by factors secreted by the tumor. Successively, ISCs can migrate into the tumor site (or a draining lymph node). Moreover, several factors produced in the tumor microenvironment may induce the conversion of $\mathrm{CD}^{+} \mathrm{T}$ cells into iTregs or drive the polarization of monocytes towards a M2-phenotype. All these phenomena lead to an inhibition of $\mathrm{CD}^{+}{ }^{+} \mathrm{T}$-helper cells and cytotoxic T lymphocytes (effector cells), resulting in a general decrease of the antitumor immune response. Theoretically, the release of ISCs from the thymus, the bone marrow, and the tumor site to the peripheral blood could reflect the immunosuppressive status of the antitumor immune response and could be of use in the real time immune monitoring of patients enrolled in therapeutic vaccine clinical trials. HPC: hematopoietic progenitor cells; CMP: committed myeloid progenitors; nTreg: thymic-derived, naturally occurring regulatory T cells; iTreg: induced or adaptive regulatory T cells; MDSC: myeloid-derived suppressor cells; TAM-M2: tumor-associated macrophages characterized by M2-polarization; CTL: cytotoxic T lymphocytes.

molecules to inhibit specific molecular pathways is also being evaluated.

Although preclinical data have shown that it is possible to break tolerance to a specific self-antigen, most clinical trials employing cancer vaccines have mostly failed to demonstrate a real advantage in terms of long-lasting clinical responses or prolonged overall survival. In an immunocompetent cancer patient, the immune system actually suppresses attacks against self-antigens, including TAAs, particularly in the tumor microenvironment. Recently, research has focused more on the suppressive component of the immune response in "breaking tolerance" and in steering the immune system toward "autoimmunity."

The major components of the suppressive compartment of the immune system are a group of heterogeneous immune cells. One of the major problems in characterizing these cells is their extreme plasticity. Cells normally committed to activating an immune response can transiently acquire suppressive characteristics. This is no doubt an important mechanism by which the immune system fine-tunes a specific immune response, balancing the number and function of immune cells involved in the process. Moreover, the heterogeneity and plasticity of this compartment of the immune system makes it difficult to define by cellular markers. A schematic representation of the specialized immune suppressive cells involved in the antitumor immune response is shown in Figure 1. A better understanding of the mechanisms that regulate the homeostasis of these suppressive cells could lead to development of more effective cancer immunotherapies and better immune monitoring of patients receiving cancer vaccines, and ultimately help to answer the question: how do cancer vaccines work? This paper will focus on the role of T-regulatory cells, myeloidderived suppressor cells, and tumor-associated macrophages in cancer immunotherapy and immune monitoring.

\section{Regulatory T Cells}

Regulatory $\mathrm{T}$ cells (Tregs) are a specialized subpopulation of $\mathrm{T}$ cells characterized by their ability to directly or indirectly suppress T-cell activation. Since their discovery in the early 1970s [4-6], the definition of Tregs has continually changed due to their extreme heterogeneity and lack of specific markers. In mice, Tregs are universally characterized by concurrent expression of CD4, CD25, and FoxP3. Although FoxP3 expression is an essential identifier 
of this population in humans, it is not sufficient, since most activated $\mathrm{CD}^{+} \mathrm{T}$ cells can transiently express FoxP3. For this reason, several different markers have been proposed to further define the phenotype of Tregs, including CD127, cytotoxic T-lymphocyte antigen 4 (CTLA-4), HLADR, glucocorticoid-induced TNFR-related protein (GITR), lymphocyte-activation gene 3 (LAG-3), CD45RA, and CD39. Unfortunately, since none of these has been demonstrated to be Treg specific [7], there is still a lack of clearly defined markers for human Tregs.

To date, there are at least 2 recognized populations of Tregs characterized by the expression of FoxP3: (1) thymic-derived, naturally occurring Tregs (nTregs) that constitutively express FoxP3, and (2) induced or adaptive Tregs (iTregs), such as $\mathrm{CD} 4{ }^{+} \mathrm{CD} 25^{-} \mathrm{T}$ cells that peripherally acquire the immunosuppressive characteristics of Tregs. Recently, it was demonstrated that these 2 lineages can be distinguished by analysis of demethylation within the FoxP3 locus [8]. To further complicate matters, at least 2 other populations of $\mathrm{CD}^{+}{ }^{+} \mathrm{T}$ cells are characterized by immunosuppressive activities: $\operatorname{Tr} 1$ and Th3. Unlike Tregs that require cell-to-cell contact to exert their suppressive activity, $\operatorname{Tr} 1$ and Th3 cells suppress mainly by the release of IL-10 and TGF- $\beta$, respectively.

The role of CTLA- 4 expression on human Tregs has been investigated for several years. CTLA-4 is expressed both within and on the surface of $\mathrm{CD} 4^{+} \mathrm{T}$ cells and Tregs, and is a negative regulator of T-cell activation. CTLA-4 counteracts the function of the costimulatory protein CD28 during antigen presentation. In fact, both molecules bind to CD80 and CD86 on antigen-presenting cells (APCs), but while CD28 transmits a stimulatory signal to $\mathrm{T}$ cells, CTLA-4 transmits an inhibitory signal, resulting in impaired T-cell activation. Expression of CTLA-4 on the surface of $\mathrm{CD}^{+}{ }^{+} \mathrm{T}$ cells is induced in response to TCR ligation, and evidently represents a mechanism of autoregulation of the immune response. In contrast, CTLA-4 is constitutively expressed on the surface of Tregs, suggesting a possible core contribution of CTLA-4 in Treg-mediated suppression [9]. Recent findings have shown that Treg-specific CTLA-4 deficiency in conditional knockout mice is associated with a profound reduction in immune suppressive capacity [10]. In addition, blockade of CTLA- 4 by specific antibodies has been shown to enhance immune responses against cancer in several clinical trials [11-14]. It is unclear, however, whether the primary target of these blocking antibodies is the effector $\mathrm{T}$ cells or the regulatory $\mathrm{T}$-cell compartment. Studies in mice have shown that anti-CTLA4 monoclonal antibody (MAb) can enhance the avidity of effector $\mathrm{T}$ cells $[15,16]$. Findings in mice expressing a chimeric CTLA-4 composed of the human extracellular domain have shown that a concomitant blockade of both compartments leads to a synergistic effect and maximal antitumor activity [17]. These data indirectly indicate the importance of balance between the stimulatory and inhibitory compartments generated during an antitumor immune response. A better understanding of these mechanisms could aid the development of novel immunotherapeutic strategies in the treatment of cancer.

\section{Tregs in Cancer Immunotherapy and Immune Monitoring}

Increased numbers and/or enhanced functionality of Tregs have been detected in the peripheral blood mononuclear cells (PBMCs), the tumor microenvironment, and in draining lymph nodes of patients with hematologic malignancies [18-20] and various types of solid tumors [21-33]. Several studies have demonstrated that Treg depletion can be used efficiently to enhance vaccine-mediated antitumor immunity in cancer patients [34-36]. A direct correlation has also been demonstrated between the frequency and function of Tregs and overall survival [35, 37-39].

A randomized placebo-controlled 43-center Phase II trial in patients $(n=125)$ with mCRPC employing a poxviralbased vaccine containing the transgenes for PSA and 3 costimulatory molecules (PSA-TRICOM) demonstrated a statistically significant $(P=.0061)$ survival advantage in the vaccine arm. In a recent study at the National Cancer Institute (NCI) employing the same vaccine, we investigated the number, phenotype, and functionality of Tregs in 32 patients with mCRPC. The median overall survival for these patients was similar to that observed in the multicenter trial of 26.6 months, with a median followup of 44.6 months; this was an improvement of 9.2 months over the median predicted survival of 17.4 months in a comparable patient population, as calculated by the Halabi nomogram [40]. Interestingly, the subpopulation of patients with a Halabipredicted survival (HPS) $>18$ months (i.e., patients with low tumor burden) seemed to benefit most from PSA-TRICOM vaccination, with an actual overall survival of $\geq 37.3$ months (median not reached) compared to an HPS of 20.9 months ( $P=.035)$ [41]. In evaluation of PBMC of these patients, we found a significant correlation between overall survival and Treg suppressive function after 3 monthly vaccinations versus prevaccination $(P=.029)$. Of patients with overall survival $>$ HPS, $80 \%$ had decreased Treg function after 3 monthly vaccinations. On the other hand, $75 \%$ of patients with overall survival < HPS showed increased Treg suppressive activity. We also investigated whether these changes in terms of Treg functionality could be related to phenotypic modifications on the surface of these cells. Based on previously published research [9], we looked at the expression of CTLA-4 as a potential marker of Treg-mediated suppression and found a significant correlation between the ratio of $\mathrm{CD} 4{ }^{+} \mathrm{CD} 25^{-}$ (effector) cells to CTLA- $4^{+}$Tregs and the overall survival of these patients. In particular, we found that the ratio increased after 3 monthly vaccinations in the subgroup of patients with overall survival $>$ HPS $(P=.029)$ and decreased after vaccination in the subgroup with overall survival $<$ HPS $(P=.027)[42]$.

Altogether, these data suggest an association between changes in Treg function after vaccination and clinical outcomes, leading to either or both of the following hypotheses: (a) these changes are a direct consequence of the postvaccination immune response, and/or (b) they reflect tumor burden and tumor escape mechanisms. Further studies are needed to address these questions. Potentially, Treg function and/or phenotype and the ratio of effector: CTLA $-4^{+}$Tregs could 
potentially be used to monitor immune function (the balance between immunostimulatory and immunosuppressive factors) in patients enrolled in clinical trials of therapeutic cancer vaccines. Analysis of Tregs in real time as part of the immune monitoring of patients could also help in identifying the subpopulation of patients who would most likely benefit from vaccine therapy versus those who would not.

\section{Myeloid-Derived Suppressor Cells}

Myeloid-derived suppressor cells (MDSCs) are a heterogeneous cell population composed mainly of myeloid progenitor cells that do not completely differentiate into mature macrophages, dendritic cells, or granulocytes. Immature bone-marrow-derived myeloid cells (IMCs) represent less than $1 \%$ of PBMCs in healthy individuals; characteristically, they retain the ability to terminally mature. In various diseases, including cancer, this subset of cells can be increased 4- to 10-fold, which is associated with partially blocked differentiation and acquisition of suppressive activity [43]. Thus, MDSCs represent critical regulators of antitumor immunity, since they can potentially inhibit both tumorspecific and nonspecific T-cell responses. MDSCs have also been shown to regulate immune responses during bacterial and parasitic infections and inflammation as well as autoimmunity [44-47]. Interestingly, a transient expansion of MDSCs has been observed after immunization with various antigens, as well as recombinant vaccinia virus expressing IL-2 [48-50].

Mouse MDSCs characteristically express markers of myeloid lineage, such as myeloid differentiation antigen (Gr1) and integrin alpha M (CD11b, also called macrophage1 [Mac-1] antigen), but they typically lack myeloid cell maturation markers. In humans, the absence of a Gr1 gene homolog has made the definition of MDSCs more challenging, and has led to the use of combinations of several different phenotypic markers, such as CD11b, CD34, CD33, CD15, CD13, CD14, IL-4R $\alpha$, and HLA-DR [48]. MDSCs can be roughly divided into 2 major subpopulations: granulocytic (PMN-MDSCs) and monocytic (MO-MDSCs). While both of these subpopulations are characterized in humans by expression of CD33, CD11b, and IL- $4 \mathrm{R} \alpha$, they differ in terms of CD14 and CD15 levels (CD14 ${ }^{-}$CD $15^{+}$for PMN-MDSCs; CD14 ${ }^{+} \mathrm{CD} 15^{-}$for MO-MDSCs).

Because of the vast heterogeneity of MDSCs, several different mechanisms of suppression have been proposed for various subpopulations of these cells [49]. A major fraction of MDSCs express high levels of intracellular arginase, an enzyme responsible for the catabolism of Larginine, a nonessential amino acid required by many cells, including $\mathrm{T}$ cells, for protein synthesis. Uptake of L-arginine by MDSCs can rapidly lead to depletion of this amino acid, resulting in a T-cell arrest in the $G_{0}-G_{1}$ phase [50]. Moreover, PMN-MDSCs can also suppress T cells by producing reactive species of oxygen, while the suppressive function of MO-MDSCs is generally mediated by inducible nitric oxide synthase (iNOS). A third potential mechanism of suppression may be related to the ability of $\mathrm{CD}_{14}{ }^{+} \mathrm{HLA}_{-} \mathrm{DR}^{-/ \mathrm{lo}}$ to induce Tregs and inhibit natural killer cells [51, 52]. Other mechanisms have also been proposed, such as sequestration of cystine (the main form of cysteine in the oxidizing extracellular environment) by MDSCs. In fact, the only form of cysteine available to $\mathrm{T}$ cells comes mainly from APCs during antigen processing and presentation, since lymphocytes lack both the enzyme (cystathionase) responsible for synthesis of this amino acid and the $\mathrm{xCT}$ chain of the $\mathrm{x}_{\mathrm{c}}{ }^{-}$cystine transporter. MDSCs have been shown to express high levels of the $\mathrm{xCT}$ chain needed to import cystine, but they lack the alanine-serinecysteine transporters needed to export cysteine. This results in sequestration of cystine from the extracellular space that ultimately leads to lower levels of cysteine available for T-cell activation. MDSCs could also act indirectly by inducing Tregs in the tumor microenvironment in the presence of IL-10 and IFN- $\gamma$, or promoting Treg expansion by acting as tolerogenic APCs [53, 54]. Finally, MDSCs could impair the homing of naïve $\mathrm{T}$ cells to draining lymph nodes by shedding Lselectin (CD62-L), operated by the ADAM metallopeptidase domain 17 (ADAM17, also called TACE, for tumor necrosis factor- $\alpha$-converting enzyme), a transmembrane glycoprotein highly and constitutively expressed on the surface of MDSCs $[49,55]$. Since ADAM17/TACE has also been related to the shedding of other important proteins involved in tumor growth and tumor escape mechanisms, such as mucin 1 and the major histocompatibility complex class I chainrelated gene-A (MICA), the constitutive expression of this "sheddase" on the surface of MDSCs may cast new light on the basic mechanisms of cancer progression, in which the accumulation of MDSCs in the tumor site could play an important role.

\section{MDSCs in Cancer Immunotherapy and Immune Monitoring}

The characteristic heterogeneity of MDSCs probably reflects the plasticity of this cell population in response to different signals received from the tumor microenvironment. In fact, each particular tumor microenvironment seems to have a unique effect on the composition of cancer-induced MDSCs, through the release of various tumor-derived factors involved in the expansion and activation of MDSCs. Cyclooxygenase 2, prostaglandins, granulocyte-macrophage colonystimulating factor (GM-CSF), macrophage CSF (M-CSF), IL-6, IL-10, vascular endothelial growth factor (VEGF), stem-cell factor, IL-3, FMS-related tyrosine kinase 3 (FLT3), and cell-expressed molecules (such as Notch) have been implicated in the expansion of MDSCs. Most of these factors trigger signaling pathways involving Janus kinase (JAK) protein family members and signal transducer and activator of transcription 3 (STAT3). IFN- $\gamma$, ligands for Tolllike receptors, IL-4, IL-13, and TGF- $\beta$ seem to be involved in the activation of MDSCs by STAT6, STAT1, and nuclear factor- $\kappa \mathrm{B}[56]$.

Recently, a subpopulation of MO-MDSCs phenotypically defined as $\mathrm{CD} 14^{+} \mathrm{HLA}^{-\mathrm{DR}^{-/ l}}$ was shown to be significantly expanded in patients with metastatic melanoma, hepatocellular carcinoma, glioblastoma, and prostate cancer [51, 5759]; increased circulating MDSCs have been correlated with 
tumor stage and metastatic spread in different types of tumors [54, 60]. Moreover, it has been demonstrated that MDSCs can be differently affected by some standard of care therapies such as sunitinib, doxorubicin-cyclophosphamide, and docetaxel and by some immunotherapies [57, 60-62]. These findings suggest a potential use for these cells in immune monitoring of cancer patients.

We have recently investigated the frequency of $\mathrm{CD}_{14}{ }^{+} \mathrm{HLA}-\mathrm{DR} \mathrm{R}^{-/ \mathrm{lo}}$ or $\mathrm{CD} 11 \mathrm{~b}^{+} \mathrm{CD} 33^{+}$cells in the PBMCs of patients with $\mathrm{mCRPC}$ before and after vaccination with PSATRICOM. Our preliminary results show that the percentage of these populations of MDSCs was significantly higher than in age-matched healthy controls. Moreover, 7 out of 10 patients with overall survival $>$ HPS showed a decreased frequency of MDSCs after vaccination. Further studies involving a range of human malignancies are obviously warranted to validate and/or expand these findings.

\section{Tumor-Associated Macrophages}

Up to $50 \%$ of a malignant tumor mass can be composed of M2-polarized tumor-associated macrophages (TAMs). Similar to Th1/Th2 polarization, monocytes in circulating blood can peripherally acquire different characteristics in response to environmental changes, assuming distinctive M1 (classical activation) or M2 (alternative activation) features. Exposure to microbial products, such as lipopolysaccharides, or IFN- $\gamma$ determines the acquisition of M1 polarization and cytotoxic functions. M1-macrophages have the ability to present antigens and activate $\mathrm{T}$ cells. They produce high levels of IL-12, IL-23, and toxic intermediates such as nitric oxide and reactive oxygen intermediates. Altogether, this activation leads to a proinflammatory response aimed at killing microorganisms and tumor cells. On the other hand, the presence of Th2-related cytokines (such as IL-4, IL-10, and IL-13) or glucocorticoids can drive the differentiation of peripheral monocytes toward an M2 phenotype, characterized by production of low levels of inflammatory cytokines and high amounts of TGF- $\beta$. M2-macrophages mainly function as scavengers, expressing surface markers such as CD206 (mannose receptor) and CD204 (scavenger receptor A) and can promote angiogenesis (they are physiologically involved in repairing and remodeling wounded/damaged tissues). It has been shown that TAMs are primarily characterized by M2-polarization and are capable of promoting tumor growth, neoangiogenesis, invasion, and metastasis by several mechanisms $[63,64]$.

\section{TAMs in Cancer Immunotherapy and Immune Monitoring}

Clinical studies have demonstrated a correlation between increased numbers of TAMs and poor prognosis for esophageal, bladder, prostate, endometrial, breast, and lung cancers [65-70]. In addition, TAMs have recently been associated with expression of VEGF and epidermal growth factor receptor in breast tumor cells [71], and have been shown to correlate with vessel density in several malignancies [72-74]. The aminobisphosphonate zoledronic acid, routinely used to prevent skeletal-related events in patients with bone metastases, has recently been shown to delay disease progression and improve survival in patients with different types of advanced cancers $[75,76]$. One of the mechanisms by which bisphosphonates prevent disease progression could be related to the inhibition of myeloid differentiation, leading to a decrease in TAMs and a shift from M2- to M1-macrophages [77].

A speculative analysis of these data could suggest a possible use of TAMs in the immune monitoring of cancer patients enrolled in clinical trials employing therapeutic vaccines. Theoretically, an efficient cancer vaccine should be able to shift an immune response against tumor toward a Th1/M1 polarization. This phenomenon could alter the frequency of TAMs in the tumor microenvironment and, consequently, in the peripheral blood of cancer patients. Thus, like Tregs and MDSCs, TAMs could likely be another suppressive cell population useful for monitoring patients in the early stage of cancer vaccine therapy.

\section{Conclusions}

Our understanding of the mechanisms that regulate suppression of immune responses has rapidly increased in recent years. In particular, the immunosuppressive role played by specific immune cells has raised questions about the importance of the balance between immunostimulation and immunosuppression in cancer immunotherapy. In many phase II/III clinical trials, boosting an antitumor immune response without counteracting the resulting immunosuppression has been shown to be only partially effective in achieving objective responses and/or prolonged overall survival [78]. Accumulating evidence suggests the potential of vaccine therapy in combination with treatments specifically aimed at depressing the number and function of immunosuppressive cells. A recent phase III trial employing ipilimumab, a monoclonal antibody that targets CTLA-4, showed an improvement in overall survival of 3.7 months in patients with advanced melanoma [79]. In addition, the receptor tyrosine kinase inhibitor sunitinib has been demonstrated to enhance antitumor immunity by reversing MDSC-mediated tumor-induced immunosuppression, and consequently improving type $1 \mathrm{~T}$-cell function in renal cell carcinoma patients [61].

Evaluation of the balance between the immunostimulatory and immunosuppressive compartments of the immune system could result in an earlier and better understanding of how a specific vaccine is working (or not) in a particular patient. The current assays used to monitor immune responses in cancer immunotherapy trials (such as enzymelinked immunospot assays, tetramer-based assays, intracellular cytokine flow cytometry, antibody tests, proliferation assays, and reverse transcription polymerase chain reaction) have shown only some usefulness as surrogate markers for clinical efficacy [80]. An assay or assays that measure the balance between immunosuppression and immunostimulation before versus after vaccination may thus fill a pressing need. 


\section{Acknowledgment}

The authors thank Bonnie L. Casey and Debra Weingarten for their editorial assistance in the preparation of this paper.

\section{References}

[1] P. W. Kantoff, C. S. Higano, N. D. Shore et al., "Sipuleucel-T immunotherapy for castration-resistant prostate cancer," New England Journal of Medicine, vol. 363, no. 5, pp. 411-422, 2010.

[2] C. Palena, S. I. Abrams, J. Schlom, and J. W. Hodge, "Cancer vaccines: preclinical studies and novel strategies," Advances in Cancer Research, vol. 95, pp. 115-145, 2006.

[3] C. Palena and J. Schlom, "Vaccines against human carcinomas: strategies to improve antitumor immune responses," Journal of Biomedicine and Biotechnology, vol. 2010, Article ID 380697, 2010.

[4] R. K. Gershon and K. Kondo, "Cell interactions in the induction of tolerance: the role of thymic lymphocytes," Immunology, vol. 18, no. 5, pp. 723-737, 1970.

[5] R. K. Gershon and K. Kondo, "Infectious immunological tolerance," Immunology, vol. 21, no. 6, pp. 903-914, 1971.

[6] R. K. Gershon, P. Cohen, R. Hencin, and S. A. Liebhaber, "Suppressor T cells," Journal of Immunology, vol. 108, no. 3, pp. 586-590, 1972.

[7] A. Corthay, "How do regulatory T cells work?" Scandinavian Journal of Immunology, vol. 70, no. 4, pp. 326-336, 2009.

[8] S. Floess, J. Freyer, C. Siewert et al., "Epigenetic control of the foxp3 locus in regulatory T cells," PLoS Biology, vol. 5, no. 2, p. e38, 2007.

[9] S. Sakaguchi, K. Wing, Y. Onishi, P. Prieto-Martin, and T. Yamaguchi, "Regulatory $\mathrm{T}$ cells: how do they suppress immune responses?" International Immunology, vol. 21, no. 10, pp. 1105-1111, 2009.

[10] K. Wing, Y. Onishi, P. Prieto-Martin et al., "CTLA-4 control over Foxp3 regulatory T cell function,” Science, vol. 322, no. 5899, pp. 271-275, 2008.

[11] F. S. Hodi, M. C. Mihm, R. J. Soiffer et al., "Biologic activity of cytotoxic $\mathrm{T}$ lymphocyte-associated antigen 4 antibody blockade in previously vaccinated metastatic melanoma and ovarian carcinoma patients," Proceedings of the National Academy of Sciences of the United States of America, vol. 100, no. 8, pp. 4712-4717, 2003.

[12] G. Q. Phan, J. C. Yang, R. M. Sherry et al., "Cancer regression and autoimmunity induced by cytotoxic $\mathrm{T}$ lymphocyteassociated antigen 4 blockade in patients with metastatic melanoma," Proceedings of the National Academy of Sciences of the United States of America, vol. 100, no. 14, pp. 8372-8377, 2003.

[13] A. Ribas, L. H. Camacho, G. Lopez-Berestein et al., "Antitumor activity in melanoma and anti-self responses in a phase I trial with the anti-cytotoxic T lymphocyte-associated antigen 4 monoclonal antibody CP-675,206," Journal of Clinical Oncology, vol. 23, no. 35, pp. 8968-8977, 2005.

[14] L. H. Camacho, S. Antonia, J. Sosman et al., "Phase I/II trial of tremelimumab in patients with metastatic melanoma," Journal of Clinical Oncology, vol. 27, no. 7, pp. 1075-1081, 2009.

[15] J. W. Hodge, M. Chakraborty, C. Kudo-Saito, C. T. Garnett, and J. Schlom, "Multiple costimulatory modalities enhance CTL avidity," Journal of Immunology, vol. 174, no. 10, pp. 5994-6004, 2005.
[16] M. Chakraborty, J. Schlom, and J. W. Hodge, "The combined activation of positive costimulatory signals with modulation of a negative costimulatory signal for the enhancement of vaccine-mediated T-cell responses," Cancer Immunology, Immunotherapy, vol. 56, no. 9, pp. 1471-1484, 2007.

[17] K. S. Peggs, S. A. Quezada, C. A. Chambers, A. J. Korman, and J. P. Allison, "Blockade of CTLA-4 on both effector and regulatory $\mathrm{T}$ cell compartments contributes to the antitumor activity of anti-CTLA-4 antibodies," Journal of Experimental Medicine, vol. 206, no. 8, pp. 1717-1725, 2009.

[18] M. Beyer, M. Kochanek, K. Darabi et al., "Reduced frequencies and suppressive function of $\mathrm{CD} 4{ }^{+} \mathrm{CD} 25^{+}$regulatory $\mathrm{T}$ cells in patients with chronic lymphocytic leukemia after therapy with fludarabine," Blood, vol. 106, no. 6, pp. 2018-2025, 2005.

[19] M. Motta, L. Rassenti, B. J. Shelvin et al., "Increased expression of CD152 (CTLA-4) by normal T lymphocytes in untreated patients with B-cell chronic lymphocytic leukemia," Leukemia, vol. 19, no. 10, pp. 1788-1793, 2005.

[20] Z. Z. Yang, A. J. Novak, M. J. Stenson, T. E. Witzig, and S. M. Ansell, "Intratumoral $\mathrm{CD} 4{ }^{+} \mathrm{CD} 25^{+}$regulatory T-cellmediated suppression of infiltrating $\mathrm{CD} 4^{+} \mathrm{T}$ cells in B-cell non-Hodgkin lymphoma," Blood, vol. 107, no. 9, pp. 36393646, 2006.

[21] E. Y. Woo, C. S. Chu, T. J. Goletz et al., "Regulatory $\mathrm{CD} 4^{+} \mathrm{CD} 25^{+} \mathrm{T}$ cells in tumors from patients with early-stage non-small cell lung cancer and late-stage ovarian cancer," Cancer Research, vol. 61, no. 12, pp. 4766-4772, 2001.

[22] L. Li, Q. G. Chao, L. Z. Ping et al., "The prevalence of FOXP3 regulatory T-cells in peripheral blood of patients with NSCLC," Cancer Biotherapy and Radiopharmaceuticals, vol. 24, no. 3, pp. 357-367, 2009.

[23] B. Karagoz, O. Bilgi, M. Gumus et al., " $\mathrm{CD} 8^{+} \mathrm{CD} 28$-cells and $\mathrm{CD} 4{ }^{+} \mathrm{CD} 25^{+}$regulatory $\mathrm{T}$ cells in the peripheral blood of advanced stage lung cancer patients," Medical Oncology, vol. 27, no. 1, pp. 29-33, 2010.

[24] L. R. Javia and S. A. Rosenberg, "CD4 ${ }^{+} \mathrm{CD} 25^{+}$suppressor lymphocytes in the circulation of patients immunized against melanoma antigens," Journal of Immunotherapy, vol. 26, no. 1, pp. 85-93, 2003.

[25] T. Sasada, M. Kimura, Y. Yoshida, M. Kanai, and A. Takabayashi, "CD4 ${ }^{+} \mathrm{CD} 25^{+}$regulatory $\mathrm{T}$ cells in patients with gastrointestinal malignancies: possible involvement of regulatory T cells in disease progression," Cancer, vol. 98, no. 5, pp. 10891099, 2003.

[26] L. S. Shen, J. Wang, D. F. Shen et al., "CD4 $4^{+} \mathrm{CD} 25^{+}-$ CD127(low/-) regulatory T cells express Foxp3 and suppress effector $\mathrm{T}$ cell proliferation and contribute to gastric cancers progression," Clinical Immunology, vol. 131, no. 1, pp. 109$118,2009$.

[27] T. J. Curiel, G. Coukos, L. Zou et al., "Specific recruitment of regulatory $\mathrm{T}$ cells in ovarian carcinoma fosters immune privilege and predicts reduced survival," Nature Medicine, vol. 10, no. 9, pp. 942-949, 2004.

[28] C. Schaefer, G. G. Kim, A. Albers, K. Hoermann, E. N. Myers, and T. L. Whiteside, "Characteristics of $\mathrm{CD} 4{ }^{+} \mathrm{CD} 25^{+}$ regulatory $\mathrm{T}$ cells in the peripheral circulation of patients with head and neck cancer," British Journal of Cancer, vol. 92, no. 5, pp. 913-920, 2005.

[29] L. Ormandy, T. Hillemann, H. Wedemeyer, M. P. Manns, T. F. Greten, and F. Korangy, "Increased populations of regulatory $\mathrm{T}$ cells in peripheral blood of patients with hepatocellular carcinoma," Cancer Research, vol. 65, no. 6, pp. 2457-2464, 2005. 
[30] P. DeLong, R. G. Carroll, A. C. Henry et al., "Regulatory T cells and cytokines in malignant pleural effusions secondary to mesothelioma and carcinoma," Cancer Biology and Therapy, vol. 4, no. 3, pp. 342-346, 2005.

[31] U. K. Liyanage, T. T. Moore, H. G. Joo et al., "Prevalence of regulatory $\mathrm{T}$ cells is increased in peripheral blood and tumor microenvironment of patients with pancreas or breast adenocarcinoma," Journal of Immunology, vol. 169, no. 5, pp. 2756-2761, 2002.

[32] A. M. Miller, K. Lundberg, V. Özenci et al., " $\mathrm{CD} 4{ }^{+} \mathrm{CD} 25^{+}$ $\mathrm{T}$ cells are enriched in the tumor and peripheral blood of prostate cancer patients," Journal of Immunology, vol. 177, no. 10, pp. 7398-7405, 2006.

[33] J. Yokokawa, V. Cereda, C. Remondo et al., "Enhanced functionality of CD $4{ }^{+} \mathrm{CD} 25$ (high)FoxP3 + regulatory T cells in the peripheral blood of patients with prostate cancer," Clinical Cancer Research, vol. 14, no. 4, pp. 1032-1040, 2008.

[34] J. Dannull, Z. Su, D. Rizzieri et al., "Enhancement of vaccinemediated antitumor immunity in cancer patients after depletion of regulatory T cells," Journal of Clinical Investigation, vol. 115, no. 12, pp. 3623-3633, 2005.

[35] M. A. Morse, A. C. Hobeika, T. Osada et al., "Depletion of human regulatory $\mathrm{T}$ cells specifically enhances antigen-specific immune responses to cancer vaccines," Blood, vol. 112, no. 3, pp. $610-618,2008$.

[36] A. J. Rech and R. H. Vonderheide, "Clinical use of anti-CD25 antibody daclizumab to enhance immune responses to tumor antigen vaccination by targeting regulatory T cells," Annals of the New York Academy of Sciences, vol. 1174, pp. 99-106, 2009.

[37] D. Mougiakakos, C. C. Johansson, E. Trocme et al., "Intratumoral forkhead box p3-positive regulatory t cells predict poor survival in cyclooxygenase-2-positive uveal melanoma," Cancer, vol. 116, no. 9, pp. 2224-2233, 2010.

[38] J. Zhou, T. Ding, W. Pan, L. Y. Zhu, A. Li, and L. Zheng, "Increased intratumoral regulatory T cells are related to intratumoral macrophages and poor prognosis in hepatocellular carcinoma patients," International Journal of Cancer, vol. 125, no. 7, pp. 1640-1648, 2009.

[39] H. Suzuki, N. Chikazawa, T. Tasaka et al., "Intratumoral CD8 T/FOXP3 cell ratio is a predictive marker for survival in patients with colorectal cancer," Cancer Immunology, Immunotherapy, vol. 59, no. 5, pp. 653-661, 2010.

[40] S. Halabi, E. J. Small, P. W. Kantoff et al., "Prognostic model for predicting survival in men with hormone-refractory metastatic prostate cancer," Journal of Clinical Oncology, vol. 21, no. 7, pp. 1232-1237, 2003.

[41] J. L. Gulley, P. M. Arlen, R. A. Madan et al., "Immunologic and prognostic factors associated with overall survival employing a poxviral-based PSA vaccine in metastatic castrate-resistant prostate cancer," Cancer Immunology, Immunotherapy, vol. 59, no. 5, pp. 663-674, 2010.

[42] M. Vergati, V. Cereda, R. Madan et al., "Circulating regulatory T-cell function and overall survival in metastatic castrationresistant prostate cancer (mCRPC) patients treated with poxviral-based vaccine," in Proceedings of the International Society for Biological Therapy of Cancer 24th Annual Meeting, Washington, DC, USA, October 2009.

[43] J. Kao, E. C. Ko, S. Eisenstein, A. G. Sikora, S. Fu, and S. H. Chen, "Targeting immune suppressing myeloid-derived suppressor cells in oncology," Critical Reviews in Oncology/Hematology, vol. 77, no. 1, pp. 12-19, 2011.

[44] M. J. Delano, P. O. Scumpia, J. S. Weinstein et al., "MyD88dependent expansion of an immature GR-1 CD11b population induces T cell suppression and Th2 polarization in sepsis,"
Journal of Experimental Medicine, vol. 204, no. 6, pp. 14631474, 2007.

[45] O. Goni, P. Alcaide, and M. Fresno, "Immunosuppression during acute Trypanosoma cruzi infection: Involvement of Ly6G (Gr1)CD11b immature myeloid suppressor cells," International Immunology, vol. 14, no. 10, pp. 1125-1134, 2002.

[46] C. Sunderkötter, T. Nikolic, M. J. Dillon et al., "Subpopulations of mouse blood monocytes differ in maturation stage and inflammatory response," Journal of Immunology, vol. 172, no. 7, pp. 4410-4417, 2004.

[47] B. Zhu, Y. Bando, S. Xiao et al., "CD11b+Ly-6C(hi) suppressive monocytes in experimental autoimmune encephalomyelitis," Journal of Immunology, vol. 179, no. 8, pp. 5228-5237, 2007.

[48] E. Peranzoni, S. Zilio, I. Marigo et al., "Myeloid-derived suppressor cell heterogeneity and subset definition," Current Opinion in Immunology, vol. 22, no. 2, pp. 238-244, 2010.

[49] S. Ostrand-Rosenberg, "Myeloid-derived suppressor cells: more mechanisms for inhibiting antitumor immunity," Cancer Immunology, Immunotherapy, vol. 59, pp. 1593-1600, 2010.

[50] P. C. Rodriguez, D. G. Quiceno, and A. C. Ochoa, "L-arginine availability regulates T-lymphocyte cell-cycle progression," Blood, vol. 109, no. 4, pp. 1568-1573, 2007.

[51] B. Hoechst, L. A. Ormandy, M. Ballmaier et al., "A new population of myeloid-derived suppressor cells in hepatocellular carcinoma patients induces CD $4^{+} \mathrm{CD} 25^{+}$Foxp $3^{+} \mathrm{T}$ cells," Gastroenterology, vol. 135, no. 1, pp. 234-243, 2008.

[52] B. Hoechst, T. Voigtlaender, L. Ormandy et al., "Myeloid derived suppressor cells inhibit natural killer cells in patients with hepatocellular carcinoma via the NKp30 receptor," Hepatology, vol. 50, no. 3, pp. 799-807, 2009.

[53] B. Huang, P. Y. Pan, Q. Li et al., "Gr- $1^{+} \mathrm{CD} 115^{+}$immature myeloid suppressor cells mediate the development of tumorinduced $\mathrm{T}$ regulatory cells and T-cell anergy in tumor-bearing host," Cancer Research, vol. 66, no. 2, pp. 1123-1131, 2006.

[54] P. Serafini, S. Mgebroff, K. Noonan, and I. Borrello, "Myeloidderived suppressor cells promote cross-tolerance in B-cell lymphoma by expanding regulatory T cells," Cancer Research, vol. 68, no. 13, pp. 5439-5449, 2008.

[55] M. K. Srivastava, P. Sinha, V. K. Clements, P. Rodriguez, and S. Ostrand-Rosenberg, "Myeloid-derived suppressor cells inhibit T-cell activation by depleting cystine and cysteine," Cancer Research, vol. 70, no. 1, pp. 68-77, 2010.

[56] D. I. Gabrilovich and S. Nagaraj, "Myeloid-derived suppressor cells as regulators of the immune system," Nature Reviews Immunology, vol. 9, no. 3, pp. 162-174, 2009.

[57] P. Filipazzi, R. Valenti, V. Huber et al., "Identification of a new subset of myeloid suppressor cells in peripheral blood of melanoma patients with modulation by a granulocytemacrophage colony-stimulation factor-based antitumor vaccine," Journal of Clinical Oncology, vol. 25, no. 18, pp. 25462553, 2007.

[58] M. P. Gustafson, Y. Lin, K. C. New et al., "Systemic immune suppression in glioblastoma: the interplay between CD14 ${ }^{+}$HLA-DRlo/neg monocytes, tumor factors, and dexamethasone," Neuro-Oncology, vol. 12, no. 7, pp. 631-644, 2010.

[59] S. Vuk-Pavlovic, P. A. Bulur, Y. Lin et al., "Immunosuppressive CD14HLA-DR monocytes in prostate cancer," Prostate, vol. 70, no. 4, pp. 443-455, 2010.

[60] C. M. Diaz-Montero, M. L. Salem, M. I. Nishimura, E. GarrettMayer, D. J. Cole, and A. J. Montero, "Increased circulating myeloid-derived suppressor cells correlate with clinical 
cancer stage, metastatic tumor burden, and doxorubicincyclophosphamide chemotherapy," Cancer Immunology, Immunotherapy, vol. 58, no. 1, pp. 49-59, 2009.

[61] J. S. Ko, A. H. Zea, B. I. Rini et al., "Sunitinib mediates reversal of myeloid-derived suppressor cell accumulation in renal cell carcinoma patients," Clinical Cancer Research, vol. 15, no. 6, pp. 2148-2157, 2009.

[62] K. N. Kodumudi, K. Woan, D. L. Gilvary, E. Sahakian, S. Wei, and J. Y. Djeu, "A novel chemoimmunomodulating property of docetaxel: suppression of myeloid-derived suppressor cells in tumor bearers," Clinical Cancer Research, vol. 16, no. 18, pp. 4583-4594, 2010.

[63] C. Guruvayoorappan, "Tumor versus tumor-associated macrophages: how hot is the link?" Integrative Cancer Therapies, vol. 7, no. 2, pp. 90-95, 2008.

[64] D. Liao, Y. Luo, D. Markowitz, R. Xiang, and R. A. Reisfeld, "Cancer associated fibroblasts promote tumor growth and metastasis by modulating the tumor immune microenvironment in a 4T1 murine breast cancer model," PLoS One, vol. 4, no. 11, Article ID e7965, 2009.

[65] N. Koide, A. Nishio, T. Sato, A. Sugiyama, and S. I. Miyagawa, "Significance of macrophage chemoattractant protein1 expression and macrophage infiltration in squamous cell carcinoma of the esophagus," American Journal of Gastroenterology, vol. 99, no. 9, pp. 1667-1674, 2004.

[66] T. Hanada, M. Nakagawa, A. Emoto, T. Nomura, N. Nasu, and Y. Nomura, "Prognostic value of tumor-associated macrophage count in human bladder cancer," International Journal of Urology, vol. 7, no. 7, pp. 263-269, 2000.

[67] I. F. Lissbrant, P. Stattin, P. Wikstrom, J. E. Damber, L. Egevad, and A. Bergh, "Tumor associated macrophages in human prostate cancer: relation to clinicopathological variables and survival," International Journal of Oncology, vol. 17, no. 3, pp. 445-451, 2000.

[68] S. Ohno, Y. Ohno, N. Suzuki et al., "Correlation of histological localization of tumor-associated macrophages with clinicopathological features in endometrial cancer," Anticancer Research, vol. 24, no. 5, pp. 3335-3342, 2004.

[69] R. D. Leek and A. L. Harris, "Tumor-associated macrophages in breast cancer," Journal of Mammary Gland Biology and Neoplasia, vol. 7, no. 2, pp. 177-189, 2002.

[70] B. C. Zhang, J. Gao, J. Wang, Z. G. Rao, B. C. Wang, and J. F. Gao, "Tumor-associated macrophages infiltration is associated with peritumoral lymphangiogenesis and poor prognosis in lung adenocarcinoma," Medical Oncology, 2010. Epub ahead of print.

[71] R. D. Leek, N. C. Hunt, R. J. Landers, C. E. Lewis, J. A. Royds, and A. L. Harris, "Macrophage infiltration is associated with VEGF and EGFR expression in breast cancer," Journal of Pathology, vol. 190, no. 4, pp. 430-436, 2000.

[72] M. Orre and P. A. W. Rogers, "Macrophages and microvessel density in tumors of the ovary," Gynecologic Oncology, vol. 73, no. 1, pp. 47-50, 1999.

[73] R. D. Leek, R. J. Landers, A. L. Harris, and C. E. Lewis, "Necrosis correlates with high vascular density and focal macrophage infiltration in invasive carcinoma of the breast," British Journal of Cancer, vol. 79, no. 5-6, pp. 991-995, 1999.

[74] A. Nishie, M. Ono, T. Shono et al., "Macrophage infiltration and heme oxygenase- 1 expression correlate with angiogenesis in human gliomas," Clinical Cancer Research, vol. 5, no. 5, pp. 1107-1113, 1999.
[75] M. Gnant, "Bisphosphonates in the prevention of disease recurrence: current results and ongoing trials," Current Cancer Drug Targets, vol. 9, no. 7, pp. 824-833, 2009.

[76] H. L. Neville-Webbe, M. Gnant, and R. E. Coleman, "Potential anticancer properties of bisphosphonates," Seminars in Oncology, vol. 37, supplement 1, pp. S53-S65, 2010.

[77] J. D. Veltman, M. E. H. Lambers, M. Van Nimwegen et al., "Zoledronic acid impairs myeloid differentiation to tumourassociated macrophages in mesothelioma," British Journal of Cancer, vol. 103, no. 5, pp. 629-641, 2010.

[78] M. Vergati, C. Intrivici, N. Y. Huen, J. Schlom, and K. Y. Tsang, "Strategies for cancer vaccine development," Journal of Biomedicine and Biotechnology, vol. 2010, Article ID 596432, 2010.

[79] F. S. Hodi, S. J. O’Day, D. F. McDermott et al., "Improved survival with ipilimumab in patients with metastatic melanoma," New England Journal of Medicine, vol. 363, no. 8, pp. 711-723, 2010.

[80] U. Keilholz, P. Martus, and C. Scheibenbogen, "Immune monitoring of T-cell responses in cancer vaccine development," Clinical Cancer Research, vol. 12, no. 7, pp. 2346s-2352s, 2006. 


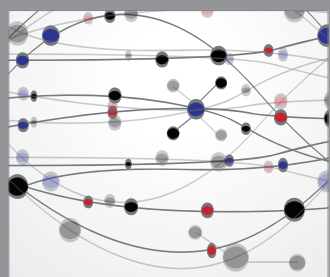

The Scientific World Journal
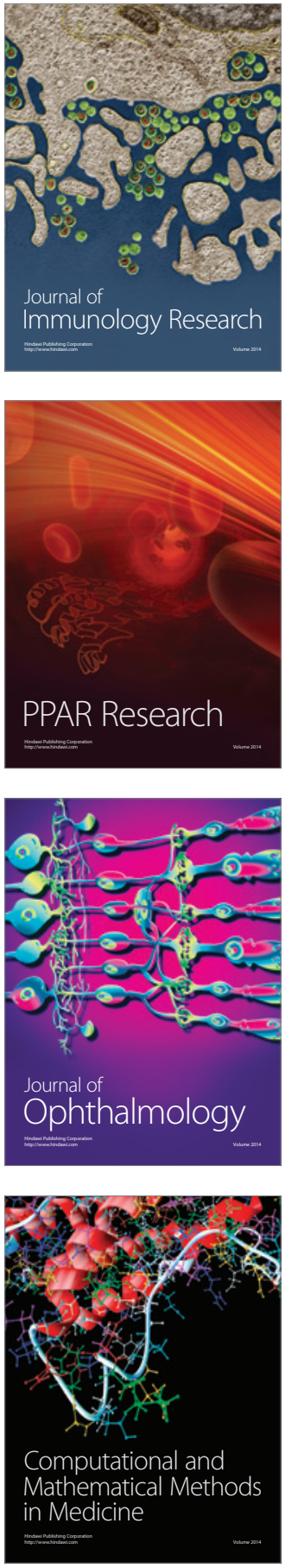

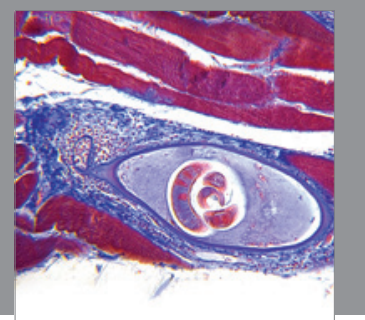

Gastroenterology

Research and Practice
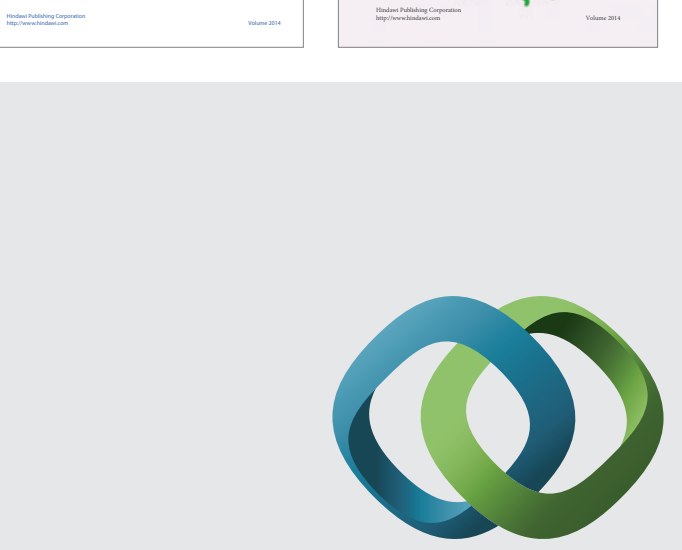

\section{Hindawi}

Submit your manuscripts at

http://www.hindawi.com
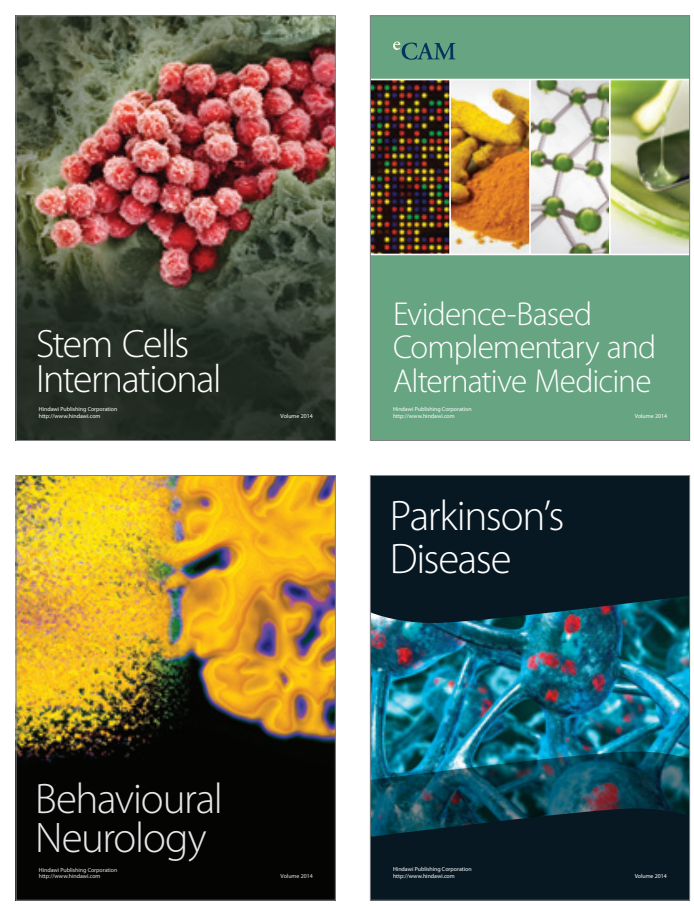

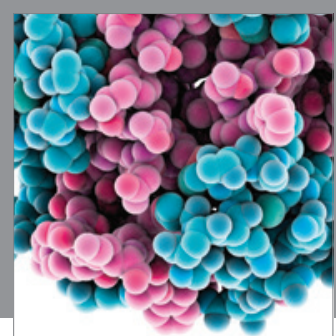

Journal of
Diabetes Research

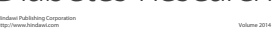

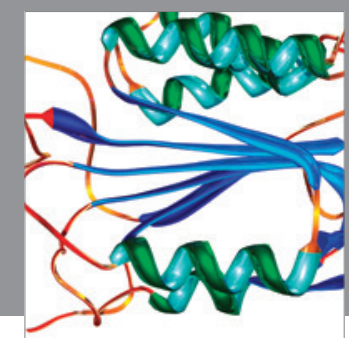

Disease Markers
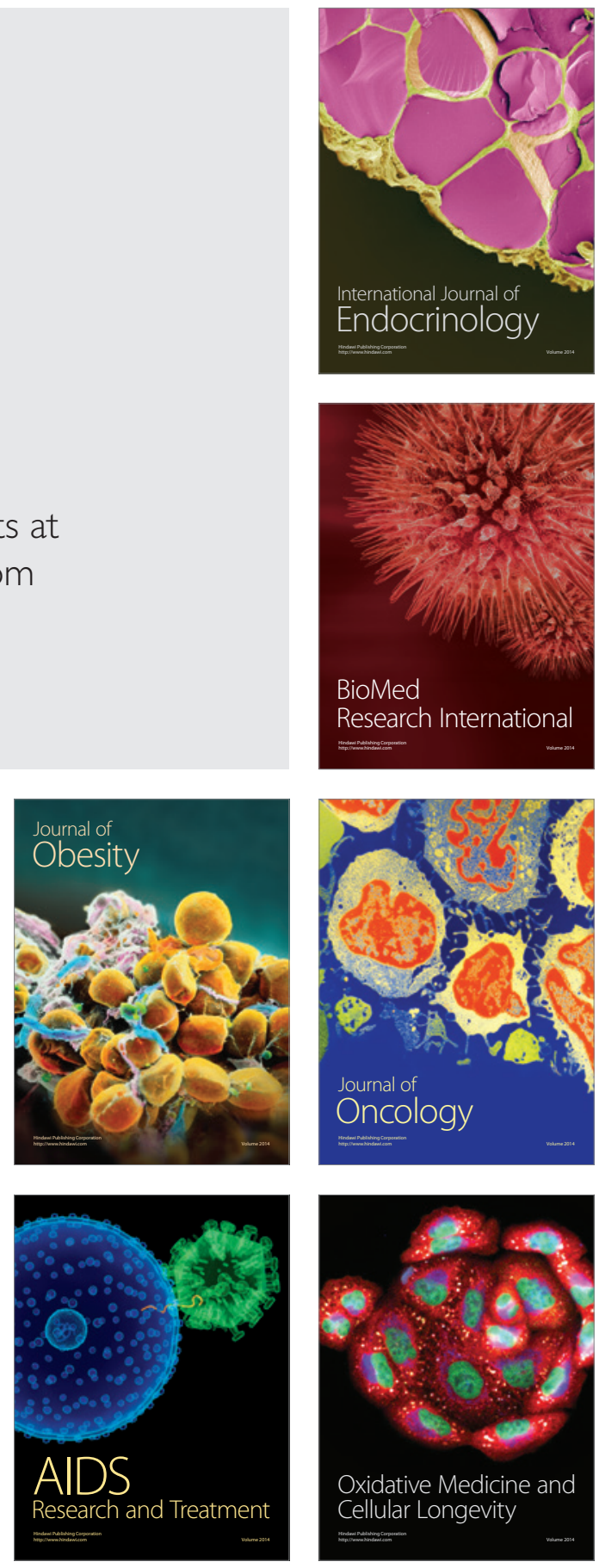